
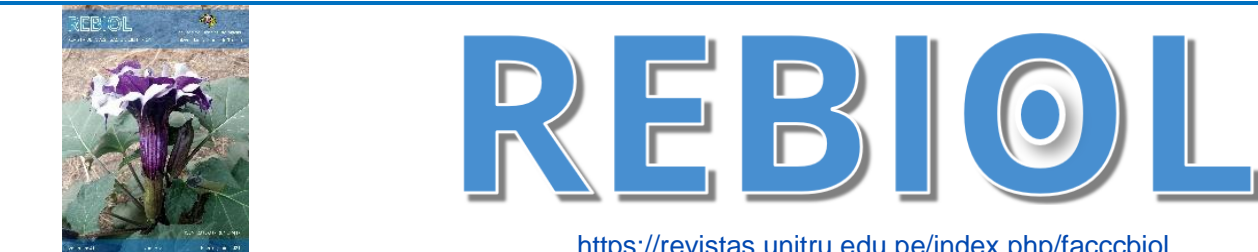

https://revistas.unitru.edu.pe/index.php/facccbiol

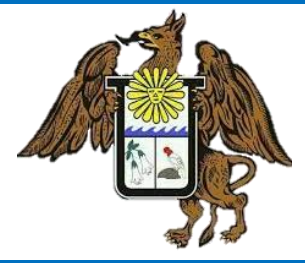

RED TRÓFICA ASOCIADA AL SAPOTE (Colicodendron scabridum) EN EL ÁREA DE CONSERVACIÓN PRIVADA LOMAS DEL CERRO CAMPANA, LA LIBERTAD, PERÚ

\title{
FOOD WEB ASSOCIATED TO THE SAPOTE TREE (Colicodendron scabridum) AT LOMAS DEL CERRO CAMPANA PRIVATE CONSERVATION AREA, LA LIBERTAD, PERU
}

\section{César Augusto Medina Tafur ${ }^{1,3}$, William Zelada Estraver ${ }^{2,3}$, Maria Elisa Seminario Rebolledo ${ }^{1,3}$, Cesia María Rodríguez Ruíz ${ }^{1}$}

\footnotetext{
${ }^{1}$ Facultad de Ciencias Biológicas, Universidad Nacional de Trujillo, Av. Juan Pablo Il s/n - Ciudad Universitaria, Trujillo, Perú.

2 Universidad Nacional Autónoma Altoandina de Tarma, Jirón Huaraz 431, Tarma, Junín, Perú.

${ }^{3}$ Museo de Zoología Juan Ormea Rodríguez - UNT, Jr. San Martín 368, Centro Histórico, Trujillo, Perú.
}

Cesar Medina Tafur:

William Zelada Estraver:

Maria Elisa Seminario Rebolledo:

Cesia Maria Rodríguez Ruíz:
(D) https://orcid.org/0000-0002-3506-849X

(iD) https://orcid.org/0000-0001-5607-5798

(iD) https://orcid.org/0000-0002-9243-3764

(D) https://orcid.org/0000-0003-4715-2848

\section{Artículo original}

Recibido: 30 de marzo 2021

Aceptado: 01 de junio 2021

\section{Resumen}

A partir de trabajo de campo y revisión bibliográfica se reporta la red trófica que está asociada al sapote (Colicodendron scabridum) en el Área de Conservación Privada Lomas del Cerro Campana (ACP LCC), en el departamento de La Libertad, norte de Perú. Nuestros resultados indican que al menos 51 especies de fauna, que incluyen 20 insectos, 5 arácnidos, 2 gasterópodos y 24 vertebrados son parte de la red trófica asociada a esta especie forestal. Esta diversidad incluye el 42,9\% de la comunidad de vertebrados del ACP LCC reportados hasta la fecha, incluyendo especies endémicas como Microlophus keopckoerum, Microlophus thoracicus, Dicrodon heterolepis, Geositta peruviana, Piezorina cinerea y Phyllotis amicus; además de otras especies de aves como: Athene cunicularia, Falco sparverius, Geranoaetus melanoleucus y mamíferos como Lagidium peruanum, Leopardus garleppi y Lycalopex sechurae. El sapote constituye un productor clave en la red trófica de este ecosistema y está seriamente amenazado, por lo que se requiere fortalecer más su protección en las partes bajas y adyacentes del ACP LCC.

Palabras clave: ACP Lomas del Cerro Campana, Colicodendron scabridum, red trófica.

\section{Abstract}

We studied the web food associated to the sapote tree (Colicodendron scabridum) at Lomas del Cerro Campana Private Conservation Area LCC PCA (La Libertad department, northern Peru) by field working and bibliography analysis. Our results show that at least 51 fauna species including 20 insects, 5 arachnids, 2 gastropods and 23 vertebrates are part of the web food asociated to this tree species. This diversity means $42,9 \%$ of vertebrates of the LCC PCA reported to date, including endemic species such as Microlophus keopckoerum, Microlophus thoracicus, Dicrodon heterolepis, Geositta peruviana, Piezorina cinerea and Phyllotis amicus; but also, other important mammal species such as Lagidium peruanum, Leopardus garleppiy Lycalopex sechurae. The sapote tree is a key producer at this area but is also threatened. Strengthening of protection actions in the lower parts of the LCC PCA and adjacent areas is highly needed.

Keywords: Colicodendron scabridum, food web, Lomas del Cerro Campana PCA.

Citar como:

Medina, C., Zelada, W., Seminario, M., \& Rodríguez, C. 2021. Red trófica asociada al sapote (Colicodendron scabridum) en el Área de Conservación Privada lomas del Cerro Campana, La Libertad, Perú. REBIOL, 41(1):35-48. 


\section{Introducción:}

El sapote Colicodendron scabridum es una especie de flora que crece como arbusto o árbol y se distribuye en el norte del Perú y sur del Ecuador entre los 0 y 2500 m de altitud (Rodríguez et al., 2007). En el Perú su distribución natural ocurre sobre los desiertos y semidesiertos, bosques secos de las vertientes occidentales y los valles interandinos bajos en los departamentos de Tumbes, Piura, Lambayeque, La Libertad, Cajamarca, Amazonas, Ancash hasta el norte de Lima (Ferreyra, 1986; Mejía et al., 1991, Rodríguez et al., 2007). En el caso de la Libertad, poblaciones de la especie han sido documentadas en el Área de Conservación Privada (ACP) Cañoncillo (Novoa, 2010), márgenes del río Chicama (Pollack et al., 2016), alrededores de Trujillo (Mejía et al., 1991), el ACP Lomas del Cerro Campana (Leiva et al., 2014) y Virú (Leiva et al., 2019).

El sapote es una especie forestal con múltiples usos para poblaciones rurales como forraje para ganado, medicina tradicional, artesanía y carpintería (Rodríguez et al., 2007, Galindo, 2012), además su goma tiene un gran potencial para la industria alimenticia y gráfica (Herz, 2007; Gonzales et al., 2013). Lamentablemente, debido al excesivo aprovechamiento de su madera y pérdida de hábitat, esta especie esta categorizada como en "Peligro Crítico" según la legislación peruana (Decreto Supremo N 043-2006$A G)$.

Esta especie tiene un importante rol en el ecosistema, ya que constituye un excelente controlador de dunas y de la erosión en el desierto (Rodríguez et al., 2007); y cumple un rol importante en la cadena trófica debido a que su polen es alimento de abejas melíferas (Saavedra et al., 2014); sus brotes, botones florales y flores son alimento de aves, incluyendo especies amenazadas como la cortarrama peruana Phytotoma raimondii (Abramonte, 2007; Pollack et al., 2016) y la pava aliblanca Penelope albipennis (Martos et al., 2009); y sus frutos, altamente energéticos y nutricionales, son alimento de ganado (Rodriguez et al., 2007), así como de mamíferos silvestres como el zorro de Sechura (Huey, 1969; Cossios, 2005) y el oso de anteojos (Appleton et al., 2018). En el ACP Lomas del Cerro Campana, las semillas del sapote son alimento también de la vizcacha Lagidium peruanum (Medina, 1996) y del zorro de Sechura Lycalopex sechurae (Sarachaga, 2002).

El ACP Lomas del Cerro Campana es la muestra representativa más importante del ecosistema de lomas en el norte del Perú (Mostacero, 1987; Sagastegui et al., 1988; Briceño et al., 1994; Rodríguez et al., 2012). El área alberga pocas especies leñosas, dentro de las que destaca el sapote que crece con hábito arbustivo, ubicándose de manera dispersa desde la base del cerro hasta los 400 m.s.n.m. (Sagastegui et al., 1988; Medina, 1996).

A pesar de que la diversidad de especies de flora de esta loma ha sido documentada por Sagastegui et al. (1998) y Leiva et al. (2014), sus vertebrados por Zelada et al. (2014) y sus artrópodos por Briceño et al. (1994), los estudios sobre las relaciones tróficas de la biota de esta loma aún son escasos. Estudios de dieta de la fauna del ACP han sido realizados para la vizcacha Lagidium peruanum (Medina, 1996), zorro de Sechura Pseudalopex sechurae (Sarachaga, 2002), águila pecho negro Geranoaetus melanoleucus (Quiroz \& Quiroz, 2012) y la lechuza de los arenales Athene cunicularia (Medina et al., 2014).

El objetivo del presente estudio fue identificar las relaciones tróficas asociadas al sapote (Colicodendron scabridum) en el ACP Lomas del Cerro Campana, y con ello incrementar el conocimiento sobre su rol ecológico y el funcionamiento en la trama trófica de esta importante comunidad lomal.

\section{Materiales y métodos}

El presente trabajo se realizó en el Área de Conservación Privada (ACP) Lomas del Cerro Campana, ubicada entre los Km 574 y 580 de la carretera Panamericana Norte; en las coordenadas geográficas Latitud Sur $7^{\circ} 57^{\prime}$ y longitud Oeste $79^{\circ} 06^{\prime}$. Estas lomas se ubican al noroeste de la ciudad de Trujillo, departamento de La Libertad, y tiene una altura de 996 m.s.n.m. (Sagástegui et al., 1988).

La caracterización de la red trófica alrededor del sapote en el ACP y su zona de influencia, se realizó mediante observaciones "in situ", así como la revisión y análisis de bibliografía sobre las relaciones tróficas de las especies incluidas en esta área.

Las observaciones in situ se hicieron durante el año 2019, cuando realizamos 12 visitas de campo al área de estudio. Cada visita fue mensual, de dos días durante los cuales se realizaron observaciones directas a partir de las 6:00 hasta las 18:00 horas. Se anotaron las observaciones de alimentación y/o interacción de las especies de vertebrados sobre un aproximado de 70 plantas de sapote. Se usaron binoculares y los puntos de observación estuvieron a una distancia de 10 a 50 m aproximadamente tratando en lo posible de no romper patrones conductuales. Se tomaron fotografías y se determinaron las especies usando guías de campo (Schulenberg et al., 2007).

En el caso de los invertebrados, se recolectaron individuos que estaban sobre las hojas, flores y frutos de los sapotes que se observaron. Los ejemplares fueron recolectados con red de golpeo y pinzas, y fueron almacenados en frascos con alcohol al 90 \% con 1 gota de glicerina. Posteriormente fueron llevados al Laboratorio de Zoología del Departamento de Ciencias Biológicas de la Facultad de Ciencias Biológicas de la Universidad Nacional de Trujillo, para su determinación con ayuda de claves taxonómicas y en algunos casos consultando a los especialistas sobre los taxa de la Universidad Nacional de Trujillo.

Para establecer la estructura trófica simultáneamente a la identificación de los organismos se establecieron por medio de consulta bibliográfica los hábitos alimenticios logrando establecer categorías y niveles tróficos para la construcción de la red. 
La determinación y presentación de los niveles tróficos de las especies asociadas al sapote se hizo siguiendo las categorías descritas por Odum (1972); Begon et al. (2006) y Marcano (2008).

\section{Resultados}

De acuerdo al hábito alimenticio, se registraron 51 especies que forman parte de la red trófica asociada a C. scabridum en el ACP Lomas del Cerro Campana y su zona de influencia. Estas especies se agrupan en ocho hábitos alimenticios: herbívoros, nectarívoros, frugívoro, insectívoros, carnívoros, omnívoros, detritívoros y coprófagos (Cuadro 1).

Cuadro 1. Especies que forman parte de la red trófica asociada al sapote (Colicodendron scabridum) en el ACP Lomas del Cerro Campana y su zona de influencia con su respectivo habito alimenticio y nivel trófico.

\begin{tabular}{|c|c|c|c|c|c|c|}
\hline $\mathrm{N}^{\circ}$ & Clase & Orden & Familia & Especie & $\begin{array}{c}\text { Habito } \\
\text { alimenticio }\end{array}$ & Nivel trófico \\
\hline 1 & \multirow{20}{*}{ 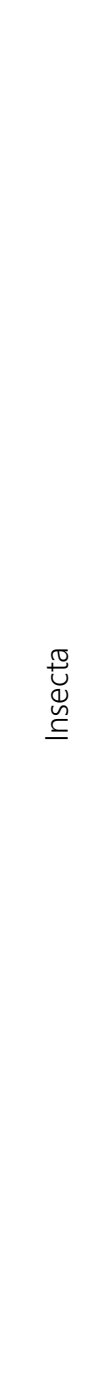 } & \multirow{6}{*}{ Coleoptera } & \multirow{3}{*}{ Coccinellidae } & Coccinellidae sp. 1 & Insectívoro & 3 \\
\hline 2 & & & & Coccinellidae sp. 2 & Insectívoro & 3 \\
\hline 3 & & & & Bothrotes sp. & Insectívoro & 3 \\
\hline 4 & & & Tenebrionidae & Parepitragus sp. & Herbívoro & 2 \\
\hline 5 & & & \multirow[b]{2}{*}{ Carabaidae } & Psammetichus sp. & Herbívoro & 2 \\
\hline 6 & & & & Calosoma abreviatum & $\begin{array}{l}\text { Detritívoro, } \\
\text { coprófago }\end{array}$ & Descomponedor \\
\hline 7 & & Diptera & Muscidae & Musca domestica & Herbívoro & 2 \\
\hline 8 & & \multirow{4}{*}{ Hemiptera } & Membracidae & Enchenopasp. & Herbívoro & 2 \\
\hline 9 & & & Pentatomidae & Nezarasp. & Herbívoro & 2 \\
\hline 10 & & & Nabidae & Nabis sp. & Insectívoro & 3 \\
\hline 11 & & & Cicadellidae & Empoasca kraemeri & Herbívoro & 2 \\
\hline 12 & & \multirow{3}{*}{ Hymenoptera } & Apidae & Apis sp. & Nectarívoro & 2 \\
\hline 13 & & & Pompilidae & Pepsinisp. & Insectívoro & 3 \\
\hline 14 & & & Vespidae & Polistes sp. & Insectívoro & 3 \\
\hline 15 & & Lepidoptera & Nymphalidae & Agraulis sp. & Nectarívoro & 2 \\
\hline 16 & & Neuroptera & Myrmeleontidae & Myrmeleon sp. & Insectívoro & 3 \\
\hline 17 & & \multirow{3}{*}{ Orthoptera } & Acrididae & Schistocerca cancellata & Herbívoro & 2 \\
\hline 18 & & & Proscopiidae & Anchotatus peruvianus & Herbívoro & 2 \\
\hline 19 & & & Acrididae & Schistocerca sp. & Herbívoro & 2 \\
\hline 20 & & Zygentoma & Lepismatidae & Lepisma gigantea & Herbívoro & 2 \\
\hline 21 & \multirow{5}{*}{ 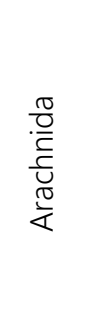 } & \multirow{4}{*}{ Araneae } & Araneidae & Argiope argentata & Insectívoro & 3 \\
\hline 22 & & & Lycosidae & Lycosa thorelli & Insectívoro & 3 \\
\hline 23 & & & Sicariidae & Loxosceles sp. & Insectívoro & 3 \\
\hline 24 & & & Theridiidae & Latrodectus mactans & Insectívoro & 3 \\
\hline 25 & & Scorpiones & Caraboctonidae & Hadruroides sp. & Insectívoro & 3 \\
\hline
\end{tabular}




\begin{tabular}{|c|c|c|c|c|c|c|}
\hline 26 & $\frac{\pi}{0}$ & & & Scutalus proteus & Herbívoro & 2 \\
\hline 27 & 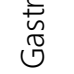 & & & Bostryx turritus & Herbívoro & 2 \\
\hline 28 & \multirow{8}{*}{ 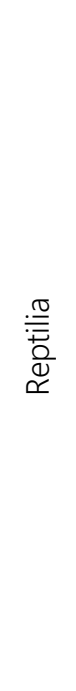 } & \multirow{8}{*}{ Squamata } & Gekkonidae & Phyllodactylus inaequalis & Insectívoro & 3 \\
\hline 29 & & & \multirow{2}{*}{ Tropiduridae } & Microlophus koepckeorum & Insectívoro & 3 \\
\hline 30 & & & & Microlophus thoracicus & Insectívoro & 3 \\
\hline 31 & & & \multirow{2}{*}{ Teiidae } & Dicrodon heterolepis & $\begin{array}{l}\text { Herbívoro, } \\
\text { insectívoro }\end{array}$ & 2,3 \\
\hline 32 & & & & Medopheos edracantha & $\begin{array}{l}\text { Herbívoro, } \\
\text { insectívoro }\end{array}$ & 2,3 \\
\hline 33 & & & Dipsadidae & Pseudalsophis elegans & $\begin{array}{l}\text { Insectívoro, } \\
\text { carnívoro }\end{array}$ & 3,4 \\
\hline 34 & & & Elapidae & Micrurus tschudii & Insectívoro & 3 \\
\hline 35 & & & Viperidae & Bothrops pictus & Carnívoro & 4 \\
\hline 36 & \multirow{12}{*}{ 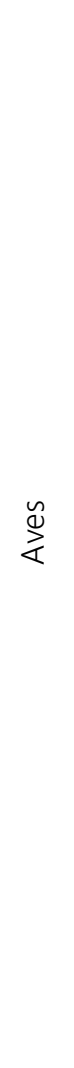 } & \multirow[b]{2}{*}{ Accipitriformes } & \multirow[b]{2}{*}{ Accipitridae } & Geranoaetus polyosoma & Carnívoro & 4 \\
\hline 37 & & & & $\begin{array}{l}\text { Geranoaetus } \\
\text { melanoleucus }\end{array}$ & Carnívoro & 4 \\
\hline 38 & & Strigiformes & Strigidae & Athene cunicularia & $\begin{array}{l}\text { Insectívoro, } \\
\text { carnívoro }\end{array}$ & 3,4 \\
\hline 39 & & Falconiformes & Falconidae & Falco sparverius & Carnívoro & 4 \\
\hline 40 & & Charadriiformes & Burhinidae & Burhinus superciliaris & $\begin{array}{l}\text { Insectívoro, } \\
\text { carnívoro }\end{array}$ & 3,4 \\
\hline 41 & & Columbiformes & Columbidae & Zenaida meloda & $\begin{array}{l}\text { Granívoro, } \\
\text { Insectívoro }\end{array}$ & 1,3 \\
\hline 42 & & \multirow{2}{*}{ Apodiformes } & Trochilidae & Amazilia amazilia & Nectarívoro & 2 \\
\hline 43 & & & Trochilidae & Rhodopis vesper & Nectarívoro & 2 \\
\hline 44 & & \multirow{4}{*}{ Passeriformes } & Hirundinidae & Pygochelidon cyanoleuca & Insectívoro & 3 \\
\hline 45 & & & Passeridae & Passer domesticus & Insectívoro & 3 \\
\hline 46 & & & Furnaridae & Geositta peruviana & Insectívoro & 3 \\
\hline 47 & & & Thraupidae & Piezorina cinerea: & Frugívoro & 2 \\
\hline 48 & \multirow{4}{*}{ 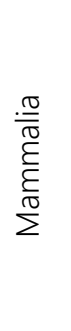 } & \multirow{2}{*}{ Carnívora } & Felidae & Leopardus garleppi & Carnívoro & 4 \\
\hline 49 & & & Canidae & Lycalopex sechurae & Omnívoro & $2,3,4$ \\
\hline 50 & & \multirow[b]{2}{*}{ Rodentia } & Chinchillidae & Lagidium peruanum & Herbívoro, & 2 \\
\hline 51 & & & Cricetidae & Phyllotis amicus & $\begin{array}{l}\text { Herbívoro, } \\
\text { insectívoro }\end{array}$ & 2,3 \\
\hline
\end{tabular}


Según el hábito alimenticio, el grupo más numeroso son los insectos herbívoros $(\mathrm{N}=10)$, seguido por los insectos insectívoros $(\mathrm{N}=7)$; mientras que los grupos menos diversos con solo una especie están representados por un insecto detritívoro-coprófago, reptil carnívoro, ave granívora, ave nectarífera, ave frugívora. mamífero carnívoro y mamífero omnívoro (Figura 1).

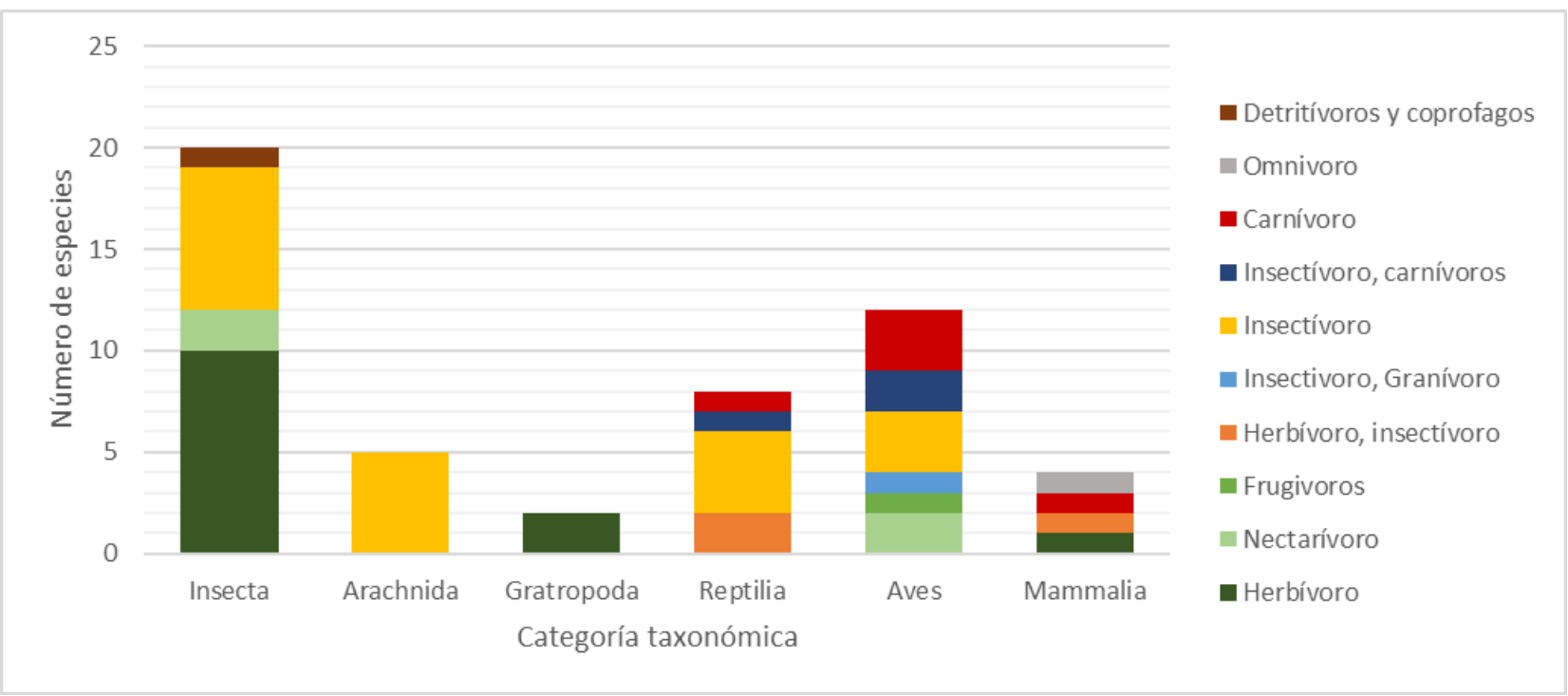

Figura 1. Número de especies según su hábito alimenticio presente en la red trófica asociada al sapote en el ACP Lomas del Cerro Campana y su zona de influencia.

Considerando al sapote como parte del primer nivel trófico (productores), las especies registradas como parte de su red trófica asociada se distribuyen en otros cuatro niveles tróficos (Figura 2): 2do nivel trófico (consumidores de primer orden), que incluye a 22 especies de insectos herbívoros y nectarívoros, gasterópodos herbívoros, reptiles herbívoros, aves nectarívoras y granívoras, y mamíferos herbívoros y omnívoros; 3er nivel trófico (consumidores de segundo orden), que incluye 26 especies de insectos y arácnidos insectívoros, reptiles insectívoros y aves insectívoras; y por último el 4to nivel trófico (consumidores de tercer orden), que incluye a 8 reptiles, aves y mamíferos carnívoros. Como descomponedores, incluye únicamente a la especie

\section{Calosoma abreviatum.}

Asimismo, se identificó 2 especies de reptiles que varían sus roles entre consumidores de primer orden $y$ eventualmente consumidores de segundo y tercer orden (lagartijas Dicrodon heterolepis y Medopheos edracantha), además existen 3 especies de vertebrados que por sus hábitos insectívoros y carnívoros pueden alternar su rol como consumidores de 3er y 4to orden (culebra elegante Pseudalsophis elegans, lechuza de los arenales Athene cunicularia y huerequeque Burhinus superciliaris). Finalmente, solo una especie de mamífero (zorro de Sechura Lycalopex sechurae), que es de hábitos omnívoros, ha sido registrado ocupando oportunistamente los 3 órdenes de consumidores. 
HABITOS

NIVEL TROFICO

ALIMENTICIOS

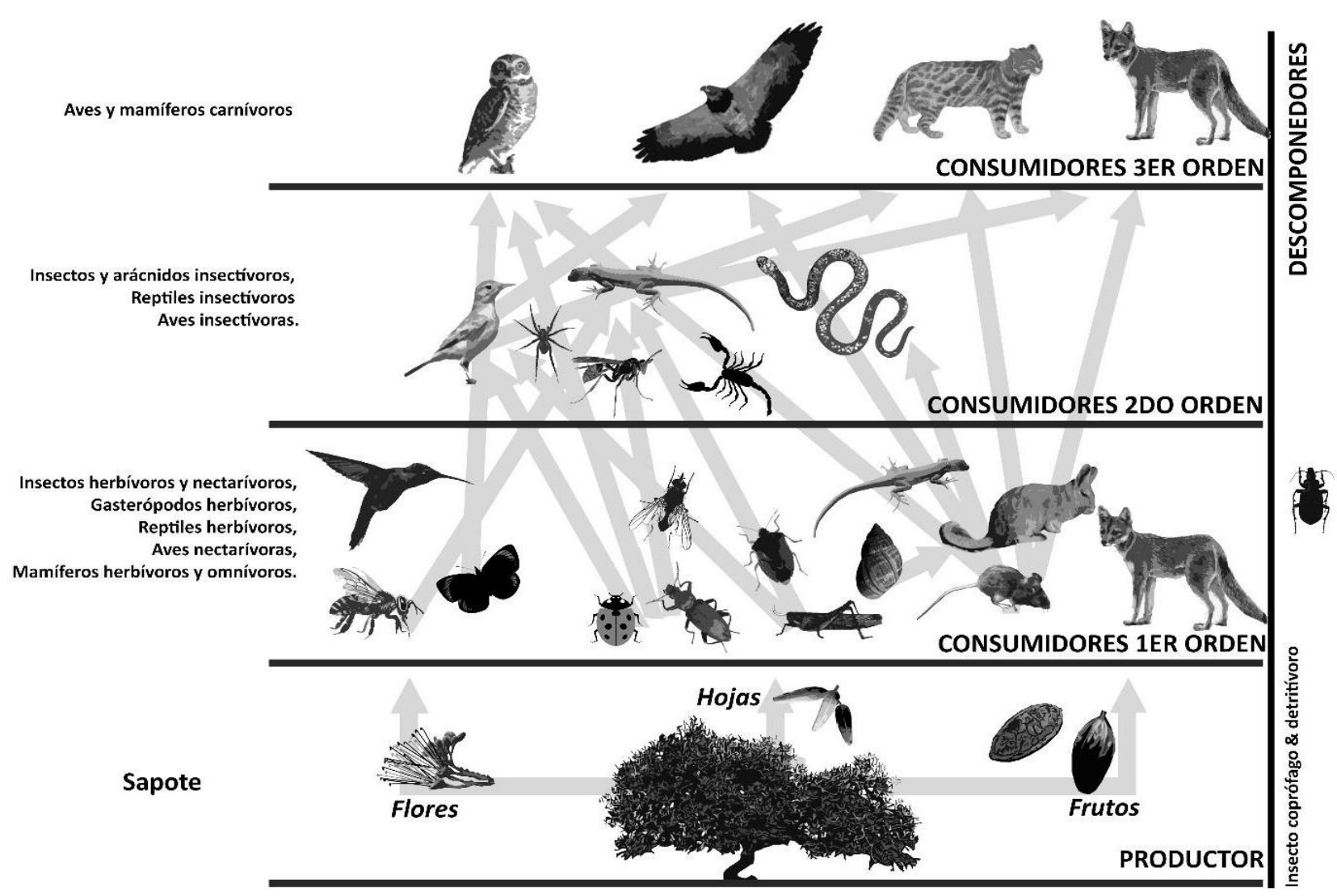

Figura 2. Esquema de los niveles tróficos y hábitos alimenticios de las especies que conforman la red trófica asociada al sapote en el ACP Lomas del Cerro Campana y su zona de influencia (elaborado por Alexander More C.).

\section{Discusión}

Las relaciones tróficas y su influencia en los ecosistemas y transformación de energía vienen siendo estudiado desde hace varias décadas desde que se introdujeron los términos de cadenas tróficas (Elton, 1927), niveles tróficos (Lindeman, 1942) y el modelo de flujo de energía universal (Odum, 1953). Actualmente los análisis de las redes y flujo trófico son realizados desde dos perspectivas: estática y dinámica, siendo el primero más descriptivo y sobre un periodo de tiempo específico, mientras que el segundo, usualmente apoyado en modelos matemáticos simula la circulación trófica y cambios estructurales y funcionales en el espacio y tiempo (Zetina-Rejón et al., 2016). Mientras que los estudios sobre la dieta de especies son relativamente más numerosos, los estudios sobre las relaciones tróficas y/o el rol que cumple una especie en estas son aún muy escasos en el Perú. En ese sentido, la presente investigación es un primer esfuerzo por caracterizar las relaciones tróficas alrededor de una especie forestal clave del bosque seco y desierto costero del norte peruano como es el sapote Colicodendron scabridum.

De acuerdo al estudio, en el ACP Lomas del Cerro Campana y su zona de influencia, el sapote es parte de la base de una red trófica y flujo energético que involucra al menos 24 especies de vertebrados, lo que constituye $42,9 \%$ de las especies de aves, mamíferos y reptiles que habitan el área (Zelada et al., 2014; Seminario et al., 2020). Si bien el sapote no es el único productor primario del $A C P$, si es muy importante reconocer que una amplia 
diversidad de vertebrados la utiliza en su dieta y su presencia genera la materia y energía para sostener elementos biológicos de todos los niveles tróficos presentes en el área desde consumidores de primer orden (nectarívoros y herbívoros) como el colibrí de oasis (Rhodopis vesper), la lagartija corredora Medopheos edracanthus, el ratón amigo (Phyllotis amicus) hasta consumidores de tercer orden o depredadores carnívoros como la lechuza de los arenales (Athene cunicularia), el cernícalo americano (Falco sparverius) y el águila pecho negro (Geranoaetus melanoleucus). La lechuza de los arenales (Athene cunicularia), es una especie además que consume los gasterópodos Scutalus proteus y Bostryx turritus, participando así en el segundo y tercer nivel trófico (Medina et al., 2014).

Por otro lado, al menos 6 especies endémicas de Perú que habitan el ACP son parte de la red trófica asociada al sapote como son las lagartijas Microlophus keopckoerum, Microlophus thoracicus, Dicrodon heterolepis, el minero peruano Geositta peruviana, el fringilo cinereo Piezorhina cinerea así como el ratón amigo Phyllotis amicus.

Adicionalmente, esta red trófica incluye 2 especies que, si bien tienen una distribución más amplia, son poco comunes en ecosistemas áridos costeros del norte peruano (Medina, 1996; García-Olaechea \& Hurtado, 2018) y en el ACP tienen poblaciones residentes como son la vizcacha Lagidium peruanum y el gato del pajonal Leopardus garleppi. Medina (1996) reporta que la semilla del sapote puede constituir hasta el $46 \%$ de la dieta de la vizcacha y L. garleppi es reportado como su principal depredador en el área.

Como particularidad de esta red trófica, debemos mencionar que el zorro de Sechura (Lycalopex sechurae) es una especie de hábitos omnívoros y oportunistas (Huey 1969; Asa \& Wallace, 1990; Cossios, 2005) y según nuestros resultados, está presente como consumidor en los 3 niveles tróficos de la red asociada al sapote en el ACP Lomas del Cerro Campana y su zona de influencia, siendo la única especie que tiene esa diversidad de hábitos alimenticios.

Entre los invertebrados, los insectos constituyen el taxa más numeroso en la red trófica asociada al sapote $(\mathrm{N}=20$, $40 \%$ de la diversidad de especies) en el ACP Lomas del
Cerro Campana. De ese total, 12 constituyen insectos herbívoros lo que representa el grupo más numeroso según sus hábitos alimenticios y por tanto un escalón clave en esta red trófica. Roubinet et al. (2018) consideran que los insectos herbívoros son un componente 'industrial' de las redes tróficas, ya que pueden condicionar la presencia de predadores que transfieren energía a los consumidores superiores; por lo tanto, su ausencia podría afectar también la estabilidad de un ecosistema.

Las relaciones tróficas alrededor del sapote en el ACP y su zona de influencia no son lineales y el flujo de energía sigue diferentes rutas (Figura 2), coincidiendo con lo reportado para otros ecosistemas (Andramunio-Acero \& Caraballo, 2012; Caraballo-Gracia et al., 2019). En este sentido, es importante destacar que es necesario más estudios sobre las interacciones tróficas entre las comunidades del ACP a fin de determinar su relevancia en la estructura y funcionamiento de este frágil ecosistema. Según Zetina-Rejón et al. (2016), uno de los principales desafíos en los estudios ecológicos es justamente demostrar como los flujos tróficos en los ecosistemas contribuyen a la estructura y función de estos, y como la explotación de algunos recursos están modificándolos. En relación a esto último, la pérdida de hábitat y los consecuentes cambios en abundancia o pérdida de especies pueden tener efectos como la contracción de la red trófica (Valladares et al., 2012) o cambios drásticos en estas (Cagnolo \& Valladares., 2011; Woodcock et al., 2013). En un bosque seco, ya se ha demostrado que un aumento en la abundancia de herbívoros y disminución de enemigos naturales son respuesta a la pérdida de la cobertura original de bosque (López, 2014).

Los ecosistemas de lomas, como el que alberga el ACP, constituyen centros de biodiversidad y recursos naturales muy importantes del desierto hiperárido de la costa peruana (López, 1967; Dourojeanni, 1987; Mostacero et al., 1987; Sagástegui et al., 1988). Lamentablemente las lomas están siendo fuertemente amenazadas, especialmente en las partes bajas que son adyacentes con poblaciones humanas (Apedjinou, 2019). En el caso del ACP Lomas del Cerro Campana se ha determinado que las principales amenazas que sufre el sapote son su aprovechamiento maderable ilegal y ocasionales incendios provocados por 
personas que ingresan al área (Medina, 1996; Pollack et al., 2020). La posibilidad de ampliación agrícola en las partes bajas y los alrededores del ACP por el proyecto Chavimochic también amenazan con destruir o fragmentar el sapotal que está dentro y rodea el ACP. El sapote (C. scabridum) constituye un productor clave sobre el cual se interrelaciona una numerosa y compleja red trófica en el ACP Lomas del Cerro Campana y su zona de influencia, por tanto, este constituiría una especie llave (Begon et al., 2006) para este ecosistema, y los efectos que puede tener la pérdida o disminución de sus poblaciones en la estructura y funcionamiento de todo este ecosistema deben ser materia de más estudios. Finalmente, y considerando que el sapote no solo tiene un rol importante en la red trófica del ACP, sino que además es un recurso promisorio para la reforestación y alimentación en el Perú (Mostacero et al., 2018), se hace necesario fortalecer la protección de la comunidad "sapotal" de las partes bajas y aledañas del ACP, así como diseñar acciones para su recuperación y expansión.

\section{Conclusiones}

El sapote (Colicodendrum scabridum) es un productor (primer nivel) de la red trófica del ACP Lomas del Cerro Campana y su zona de influencia sobre el cual se interrelacionan al menos 51 especies de fauna, incluyendo 20 insectos, 5 arácnidos, 2 gasterópodos y 24 vertebrados, esto último representa el $42,9 \%$ de esta comunidad en el ACP.

La biodiversidad que es parte de la red trófica asociada al sapote se distribuye en 7 hábitos alimenticios y 4 niveles tróficos, incluyendo los 3 niveles de consumidores.

El sapote constituye un productor clave y una especie llave para el ecosistema del ACP y su zona de influencia; lamentablemente amenazado, por lo que urgen acciones para su protección, recuperación.

\section{Agradecimientos}

Agradecemos al Blgo. Alexander More Cahuapaza por la elaboración de la Figura 2.

\section{Contribución de los autores}

CMT (1) la concepción y el diseño del estudio, los registros, fotografías, redacción del borrador del artículo y la revisión crítica del contenido y la aprobación final. WZE,
CRR y MSR (2) los registros, fotografías, redacción del borrador del artículo y la revisión crítica del contenido y la aprobación final.

\section{Conflicto de intereses}

No existe conflicto de intereses en la redacción, revisión y publicación de este manuscrito.

\section{Referencias bibliográficas}

Abramonte, C. (2007). Conducta reproductiva y dieta de Phytotoma raimondii (Taczanowski, 1883) "cortarrama peruana" en el bosque seco de Talara, Piura. Tesis para optar el grado de Biólogo. Universidad Nacional de Piura.

Apedjinou, K. (2019). Impacto del crecimiento urbano en la alteración y degradación del ecosistema de las lomas de Villa María del Triunfo. Tesis de Maestría en Ecología y Gestión Ambiental. Universidad Ricardo Palma.

Andramunio-Acero, C. y Caraballo, P. (2012). Análisis de las relaciones tróficas en un lago de inundación de la Amazonia Colombiana. Rev. Colombiana Cienc Anim, 4(1), 102-120.

Appleton, R. D., Van Horn, R. C., Noyce, K.V., Spady, T.J., Swaisgood, R.R. y P. Arcese. (2018). Phenotypic Plasticity in the Timing of Reproduction in Andean Bears. Journal of Zoology, 305(3), 196-202.

Asa, C y Wallace, M. (1990). Diet and activity pattern of the sechuran desert fox (Dusicyon sechurae). Journal of mammalogy, 71, 69-72.

Begon, M., Townsed, C. y Harper, J. (2006). Ecology: From Individuals to Ecosystems. Fourth Edition. BlackWell Publishing. pp. $579-583$

Briceño, J., E. Rodríguez; L. Pollack. y C. Vergara. (1994). Importancia natural y cultural del Cerro Campana: estado actual y perspectivas. Actas de la Segunda Jornada de Investigación en Ciencias Biológicas, pp. 402-406.

Cagnolo, L. y Valladares,G. (2011). Fragmentación del hábitat y desensamble de redes tróficas. Ecosistemas, 20 (2), 68-78.

Caraballo-Gracia, R., Martinez-Prada, S. y Salcedo-Díaz. A. (2019). Relaciones tróficas en un agroecosistema de la región Sabanas, Sucre, Colombia. Pastos y Forrajes, 42 (2), 152-160.

Cossios, D. (2005). Dispersión y variación de la capacidad de germinación de semillas ingeridas por el zorro costeño (Lycalopex sechurae) en el Santuario Histórico Bosque de Pomac, Lambayeque. Tesis de Magister en Zoología. Universidad Nacional Mayor de San Marcos.

Decreto Supremo Nº43 (2006). Aprueban categorización de especies amenazadas de flora silvestre. En: Normas Legales. Diario Oficial "El Peruano". Ministerio de Agricultura. Lima.13 de Julio de 2006, pp. 323527-323539.

Dourojeanni, M. (1987). Recursos Naturales y Desarrollo en la Libertad. Boletín de Lima, 53, 69 - 74. Lima - Perú.

Elton C. (1927). Animal ecology. Macmillan Co., New York. 209p.

Ferreyra, R. (1986). Flora y Vegetación del Perú. En Gran Geografía del Perú. Vol 2. Edit. Juan Mejía Baca. Edic. Manfer. Barcelona, España.

Galindo, F. 2012. Análisis del fruto de Capparis scabrida H.B.K. (sapote), en función de las características del árbol, proveniente de la comunidad San Julián de Motupe, Motupe, Lambayeque. Tesis de Ingeniero Forestal. Universidad Nacional Agraria La Molina. Lima, Perú.

García-Olaechea, A. y Hurtado, C. (2018). Assessment of the current distribution and human perceptions of the Pampas cat Leopardus colocolo in northern Peru and southern Ecuador. Oryx, 52(3), 587-590.

Gonzales, E.; K. Begazo, D. Guzman, G. Chire, y Ascencios, D. (2013). El árbol del sapote (Capparis scabrida) como recurso 
forestal. Boletín del Sub-Proyecto Sapote, Proyecto Desarrollo de cadenas de valor para la conservación de la biodiversidad y mejora de vida rural. Compartir mentes, cambiar vidas Vlir-Uos - Bélgica. Cooperación para el Desarrollo de Bélgica. Universidad Nacional Agraria La Molina.

Herz, K. (2007). Análisis Físico-Químico de la Goma Exudada de la Especie Sapote Capparis scabrida H.B.K., Proveniente de los Bosques Secos de Lambayeque. [Tesis de pregrado, Universidad Nacional Agraria La Molina. Lima, Perú].

Huey, R. (1969). Winter diet of the Peruvian desert fox. Ecology, 50, 1089-1091.

Leiva, S., M. Cruz, G. Gayoso, L. Chang, M.O. Dillon y Quipuscoa. V. (2014). Diversidad Florística de la Loma Cerro Campana, Provincia Trujillo, Departamento La Libertad-Perú. Arnaldoa, $21(1), 187-220$.

Leiva, S.; E. Rodríguez; L. Pollack; J. Briceño; G. Gayoso; L. Chang y Guerrero. A. (2019). Diversidad de flora, fauna y disponibilidad hídrica en el centro poblado Caray, distrito, provincia Virú, Perú. Arnaldoa, 26 (1), 223-276.

Lindeman R. (1942). The trophic-dynamic aspect of ecology. Ecology, 23, 157-176.

López, A. (1967). Se propone declarar Parques Nacionales a las Lomas más importantes de la Costa Peruana. Rev. Facultad de CC.BB. de la Univ. Nac. de Trujillo, I (3), 88.

López, A. (2014). Interacciones insecto-planta en una leguminosa del bosque seco tropical bajo de tres coberturas con diferente perturbación: Coello, Tolima, Colombia. [Tesis de pregrado, Pontificia Universidad Javeriana. Bogotá].

Marcano, J. (2008). Niveles tróficos. Ediciones Mundi- Prensa. Madrid.

Martos, J., Scarpati, M., Rojas, C., y Delgado, J. (2009). Fenología de algunas especies que son alimento para la Pava Aliblanca Penelope albipennis. Revista Peruana de Biología, 15(2), 5158.

Mejía, F., Medina, D y Mostacero, J. (1991). Sapote, prodigioso recurso de la Costa Norte del Perú. Boletín de Lima, 73, 4356.

Medina, C (1996). Presencia, alimentación y aspectos etológicos de Lagidium peruanum Meyen 1833 "vizcacha"en la Loma del "Cerro Campana". [Tesis de pregrado, Universidad Nacional de Trujillo].

Medina, C., Zelada, W. y Pollack, L. (2014). Estructura alimenticia de la lechuza de los arenales, Athene cunicularia, en el Cerro Campana, La Libertad, Perú. 2011. Rebiol, 34 (2), 73-82.

Mostacero, J., Ramírez., Mejía, F., Peláez, F y Medina, D. (1987). Variación Estacional de la Vegetación de las Lomas de la Prov. de Trujillo. Rebiol, 7 (1-2), 58-79. p.

Mostacero, J., De La Cruz, J y L. Taramona (2018). Características germinativas de Colicodendron scabridum (Kunth) Seem.: Recurso promisorio para la reforestación y alimentación en el Perú. Rev. Investig. Univ. Le Cordon Bleu, 5(1), 117-131

Novoa, Z. (2010). Plan Maestro del Área de Conservación Privada Bosque Natural El Cañoncillo 2011- 2015. Lima, PE, CIGAPUCP. 67 p.

Odum, E. (1953). Fundamentals of ecology. Philadelphia: W. B. Saunders Company.

Odum, E. (1972). Ecología. Tercera Edición. Nueva Editorial Interamericana.

Pollack, L., Rodríguez, E., Alvitez, E y Huamán, E. (2016). Nuevo registro y hábitos alimentarios de Phytotoma raimondi (Passeriformes: Cotingidae) en la provincia Gran Chimú, región La Libertad, Perú. Arnaldoa, 23 (2), 649 - 664.

Pollack, L., Rodríguez, E., Leiva, S., Saldaña, IE., Alvitez, E., Briceño y Gayoso.G. (2020). Amenazas y desastres antrópicos frecuentes en el Área de Conservación Privada (ACP) Lomas
Cerro Campana (provincias Trujillo y Ascope, región La Libertad, Perú). Arnaldoa, 27(1), e53-e63.

Quiroz, C. y Quiroz, C. (2012). Aspectos etológicos y ecológicos de Geranoaetus melanoleucus (Swann, 1922) "Águila de Pecho Negro" en el Cerro Campana. Pueblo Cont, 23(2), 308329.

Rodríguez, E., Liza., Martínez, B., Pollack, L., Leiva, S., Zapata M y Mora, M. (2012). Loma el Cerro Campana, Patrimonio Natural y Cultural de la Región La Libertad. Pueblo Cont, 23(2), 330336.

Rodríguez, E., Bussmann, R., Rainer W., Arroyo, S., López, S. y J. Briceño. (2007). Capparis scabrida (Capparaceae) una especie del Perú y Ecuador que necesita planes de conservación urgente. Arnaldoa, 14(2), 269-82.

Roubinet, E., Jonsson, T., Malsher, G., Staudacher, K., Traugott, M., Ekbom y Jonsson, M. (2018). High redundancy as well as complementary prey choice characterize generalist predator food webs in agroecosystems. Scientific reports, 8 (1), 8054.

Saavedra, D., Aguinaga, F., Rojas, C y Delgado, G. (2014). Analysis of pollen loads collected by honey bees (Apis mellifera L.) from Lambayeque province (Peru): Botanical origin and protein content. Journal of Global Biosciences, 3, 285-298

Sagastegui, A., Mostacero, J y López, S. (1988). Fitoecología del "Cerro Campana". Provincia de Trujillo. Bol. Soc. Bot. La Libertad, 14(1-2), 1-83.

Sarachaga, L. (2002). Aspectos etológicos y ecológicos de Pseudalopex sechurae Thomas 1900, Zorro Costero en la Loma del Cerro Campana, Trujillo; entre agosto 1998 y mayo 1999. [Tesis de pregrado, Universidad Nacional de Trujillo].

Schulenberg T. S., Stotz, D. F. Lane, D. F. O'Neill, J. P \& Parker, T. A. (2007). Birds of Peru. Princeton Univ. Press. Princeton, New Jersey.

Seminario, M., Zelada, W., Medina, C y Sánchez, V. (2020). Registro de Asio flammeus (Aves: Strigidae) para el Área de Conservación Privada Lomas del Cerro Campana, La Libertad. REBIOL, 40 (2), 318 - 325.

Valladares, G., Cagnolo L y Salvo, A. (2012). Forest fragmentation leads to food web contraction. Oikos, 121, 299-305.

Woodcock, P., Edwards DP., Newton RJ., Vun Khen C., Bottrell SH y Hamer, KC. (2013) Impacts of Intensive Logging on the Trophic Organisation of Ant Communities in a Biodiversity Hotspot. PLOS ONE, 8(4), e60756.

Zelada, W., Polláck, L., Medina, C.\& Castillo, H. (2014) Vertebrados del sistema lomal Cerro Campana, Trujillo, Perú. Arnaldoa, 21 (1), 221-240.

Zetina-Rejón, M., Brown-Peterson, N., Del Monte, P., CruzEscalona, V y Peterson, M. (2016). Trophic circulation in ecosystems. Ecosistemas y Recursos Agropecuarios, 3(8), 279-291. 


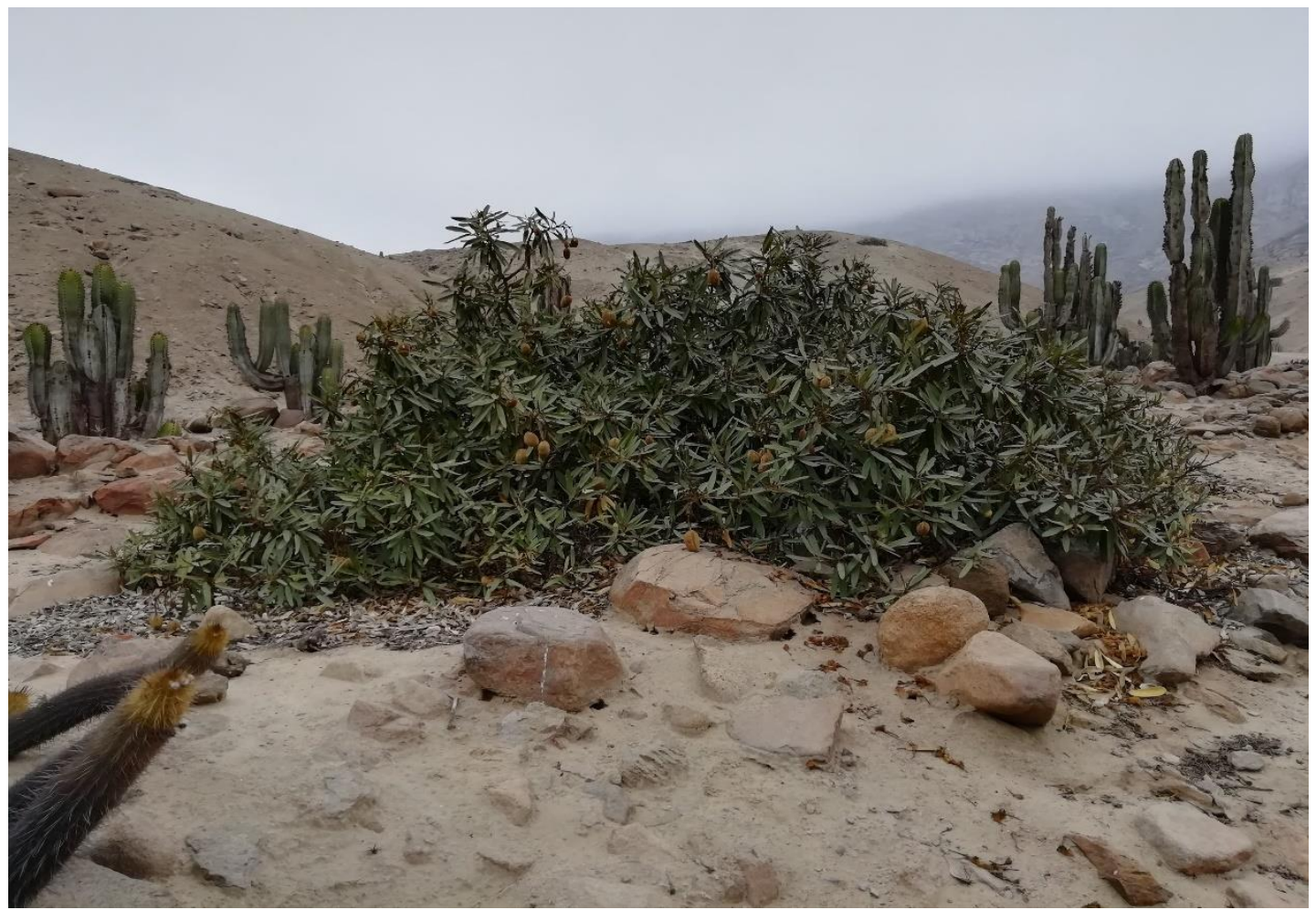

Figura. 3. Ejemplar de Colicodendron scabridum, integrante del primer nivel trófico (productores) en el ACP Lomas del Cerro Campana, durante el 2019. 


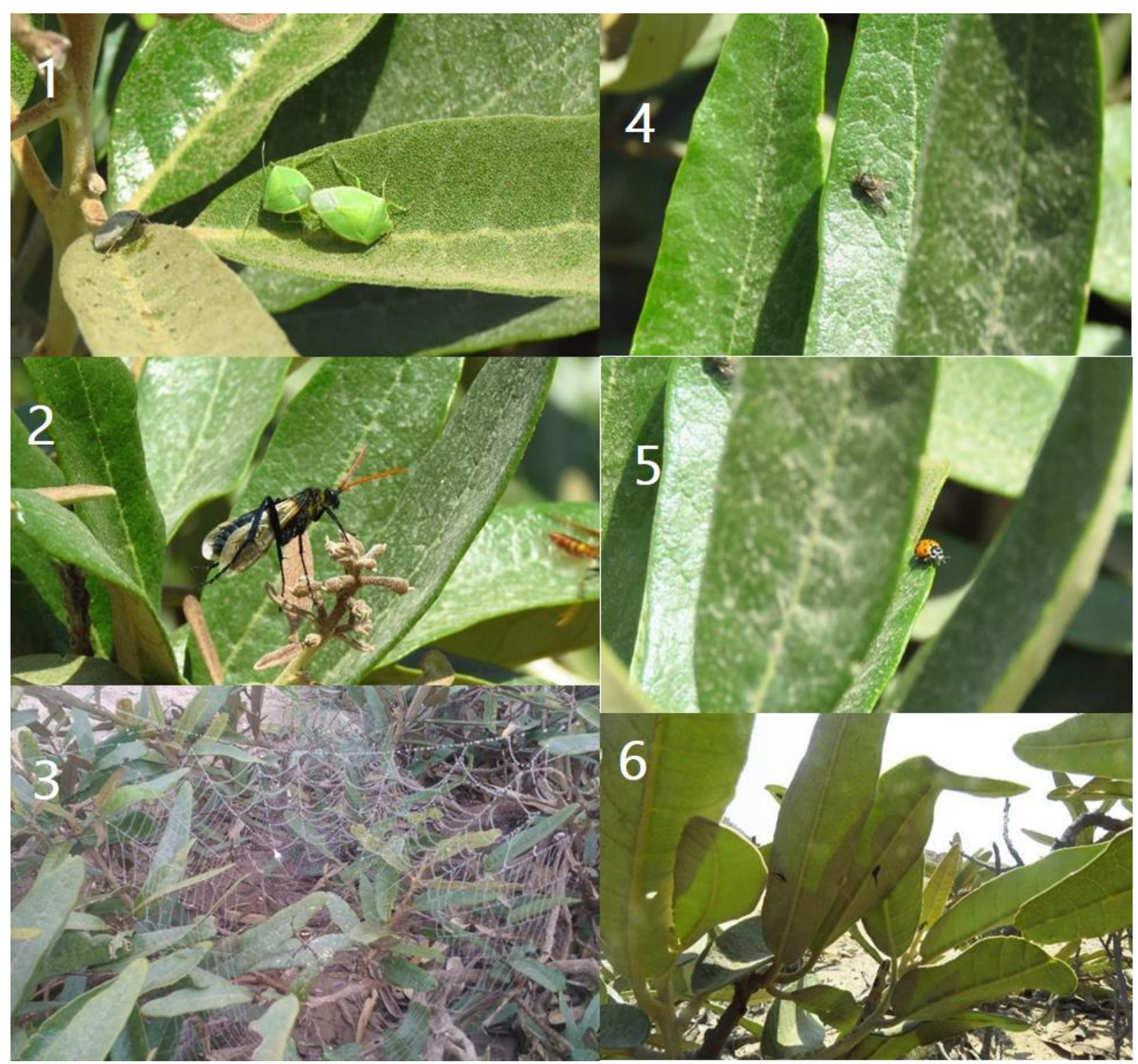

Figura 4. Ejemplares de insectos:(1) Nezara sp. (2) Pepsini sp. (3) Argiope argentata (4) Musca domestica (5) Coccinellidae (6) Enchenopa sp. presentes en las hojas de C. scabridum "sapote", en el Área de Conservación Privada Lomas del Cerro Campana, durante el 2019. 


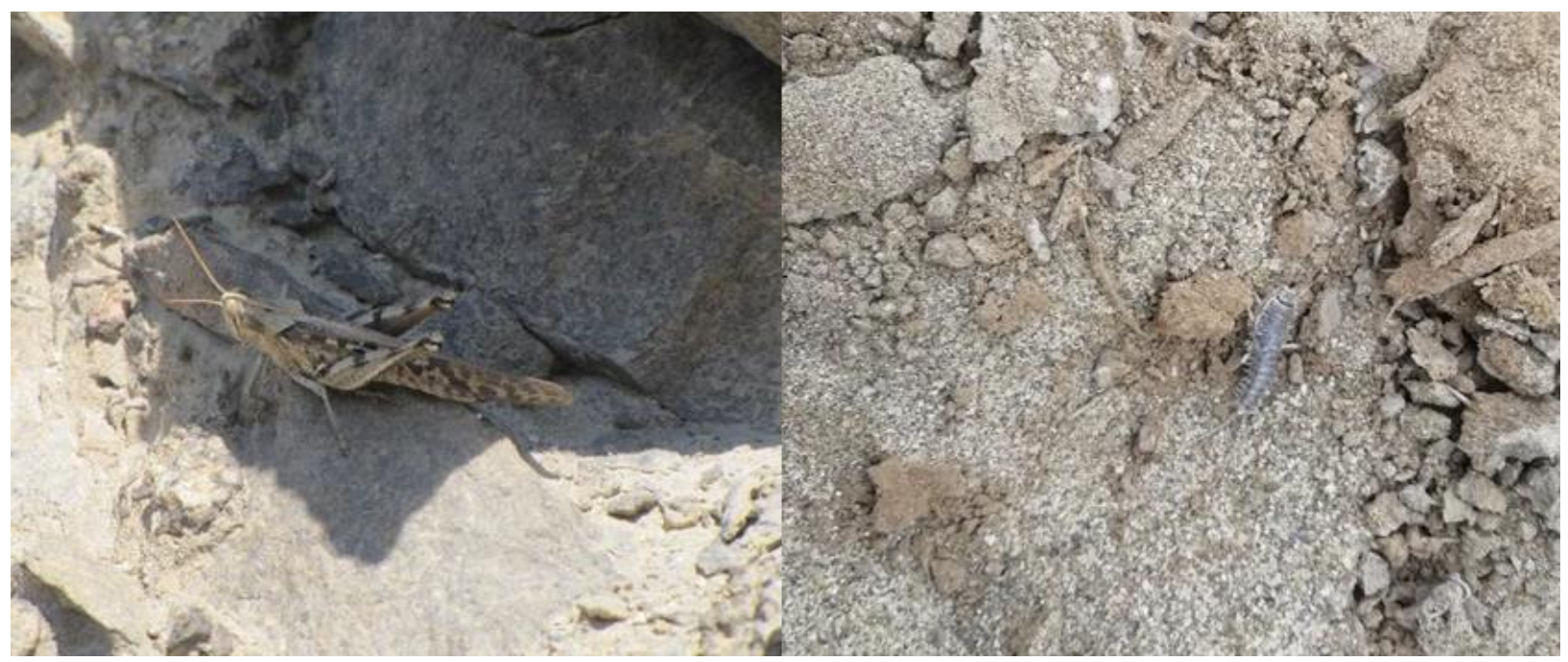

Figura 5. Ejemplar de Schistocerca cancellata y de Lepisma gigante presentes en las hojas de C. scabridum "sapote", en el Área de Conservación Privada Lomas del Cerro Campana. durante el 2019.

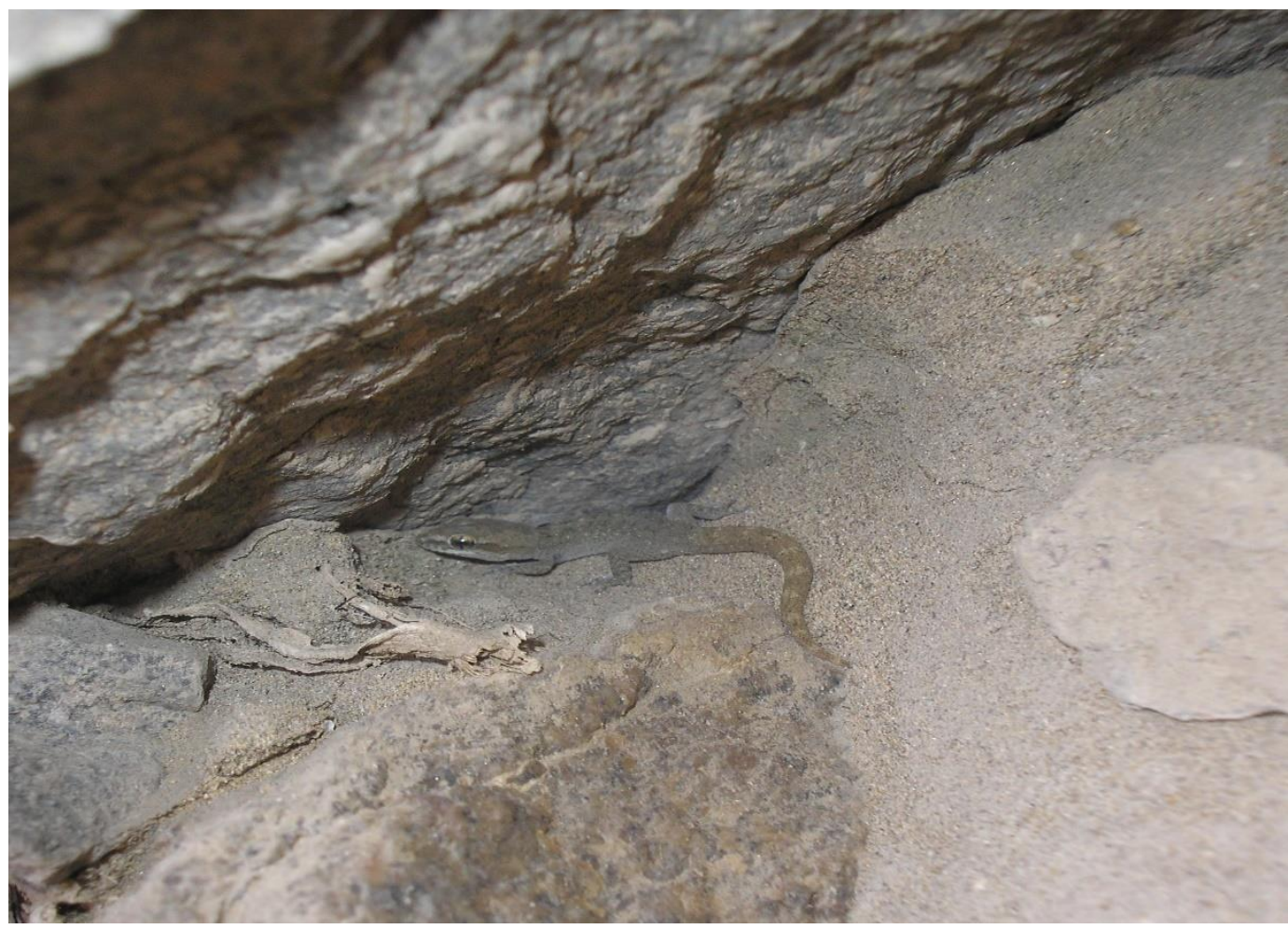

Figura 6. Ejemplar de saltojo Phyllodacty/us inaequalis integrante del segundo orden, consumidor de insectos asociados a C. scabridum "sapote" integrante del tercer nivel trófico en el Área de Conservación Privada Lomas del Cerro Campana durante el 2019. 


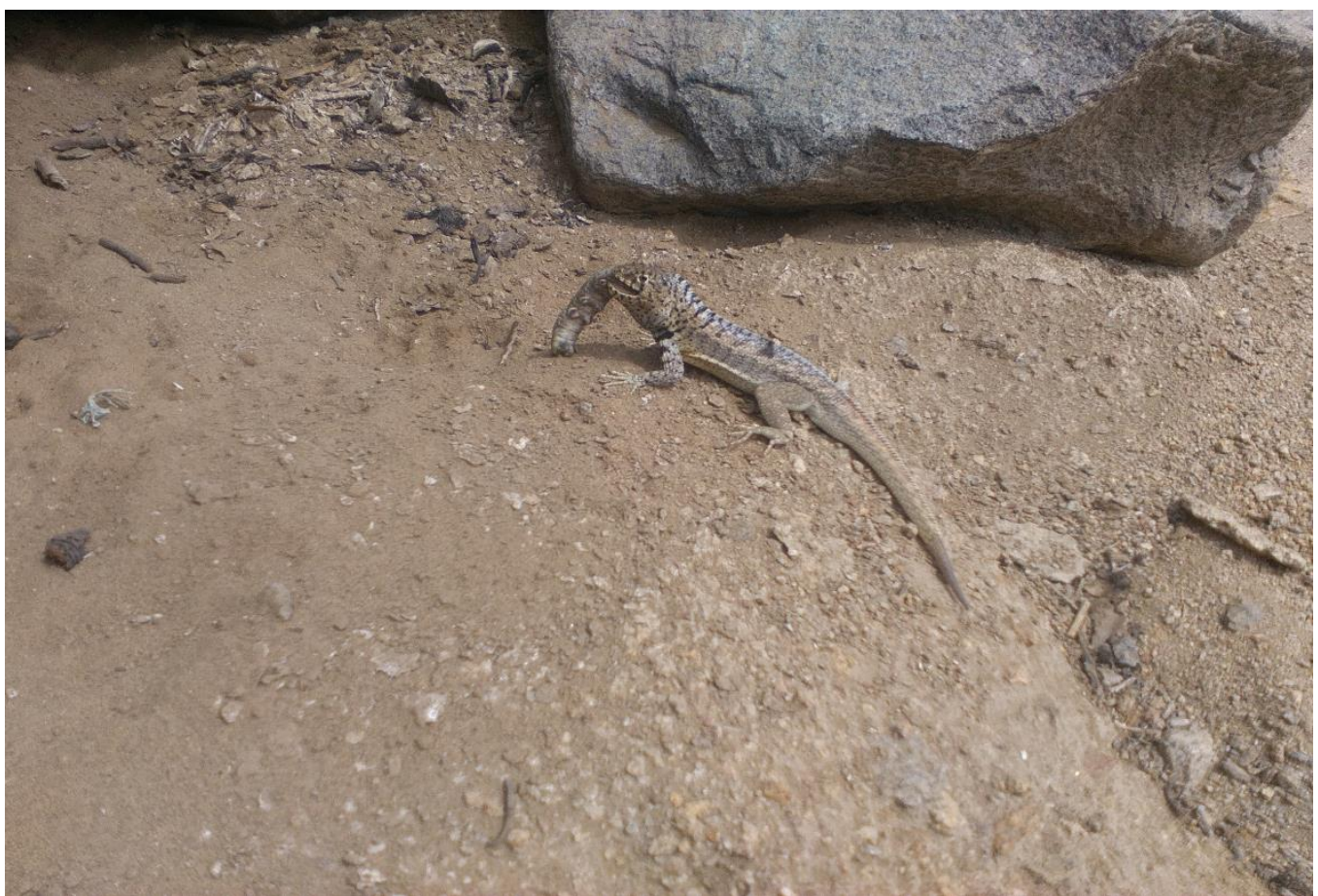

Figura 7. Ejemplar de Microlophus keopckoerum integrante del segundo orden consumiendo una larva de Lepidóptero integrante del primer orden en el Área de Conservación Privada Lomas del Cerro Campana durante2019.

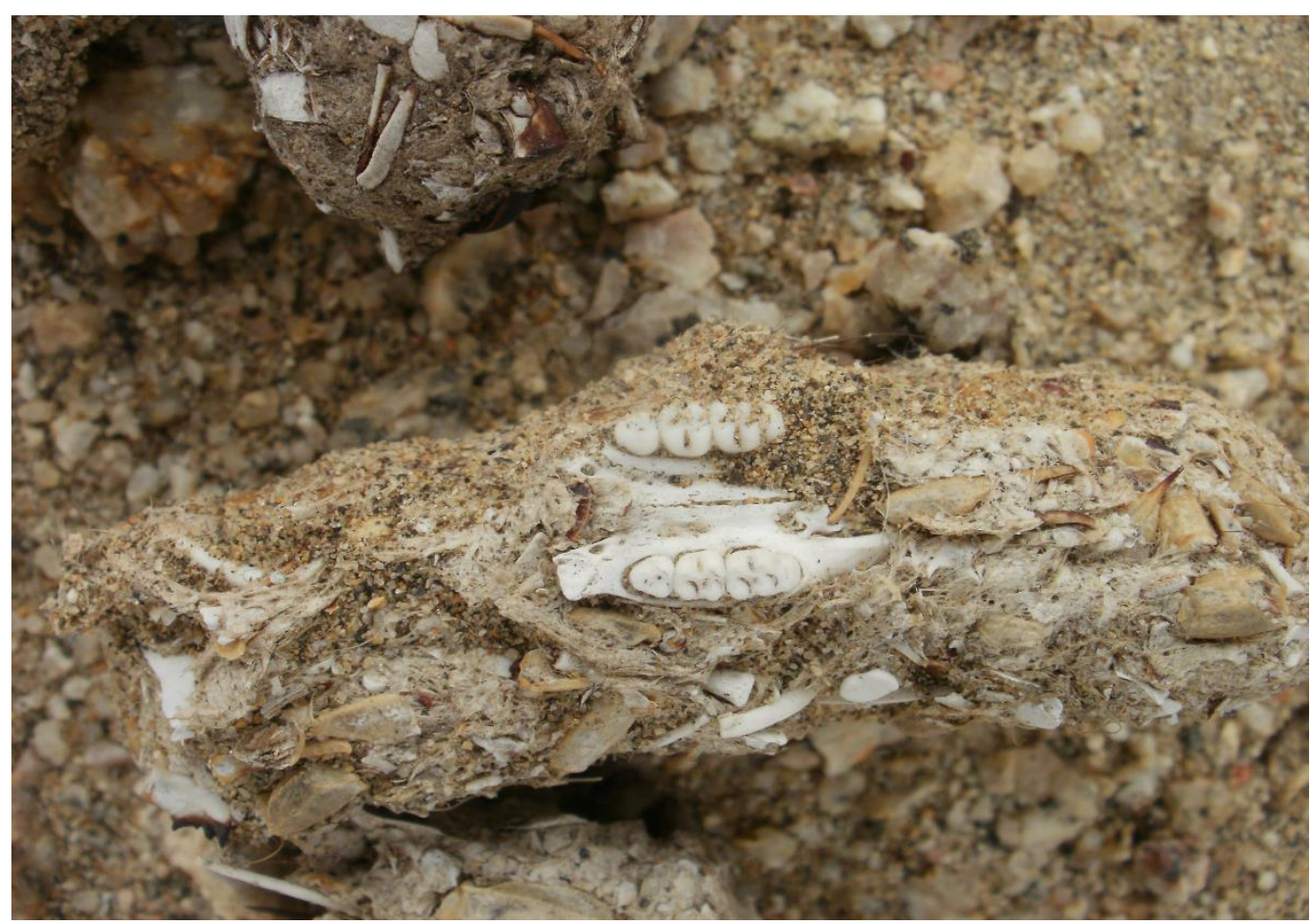

Figura 8. Egagropila de Athene cunicularia integrante del tercer orden con restos óseos de Phyllotis amicus y estructuras de insectos (Schistocerca cancellata) y arácnidos (Hadruroides sp) y en el Área de Conservación Privada Lomas del Cerro Campana. durante el 2019. 


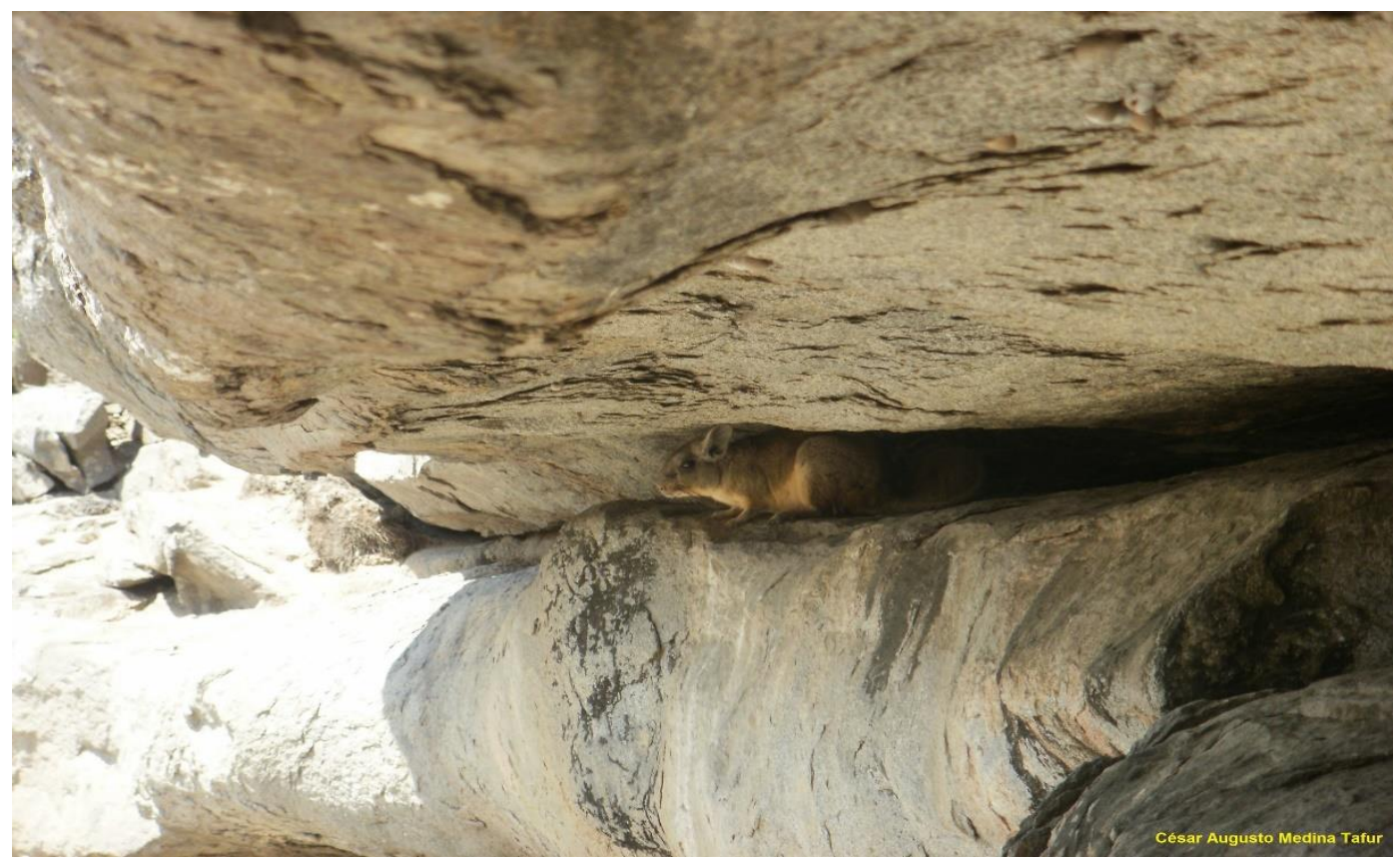

Figura. 9. Ejemplar de vizcacha Lagidium peruanum consumidor de C. scabridum "sapote" integrante del primer nivel trófico en el Área de Conservación Privada Lomas del Cerro Campana durante el 2019

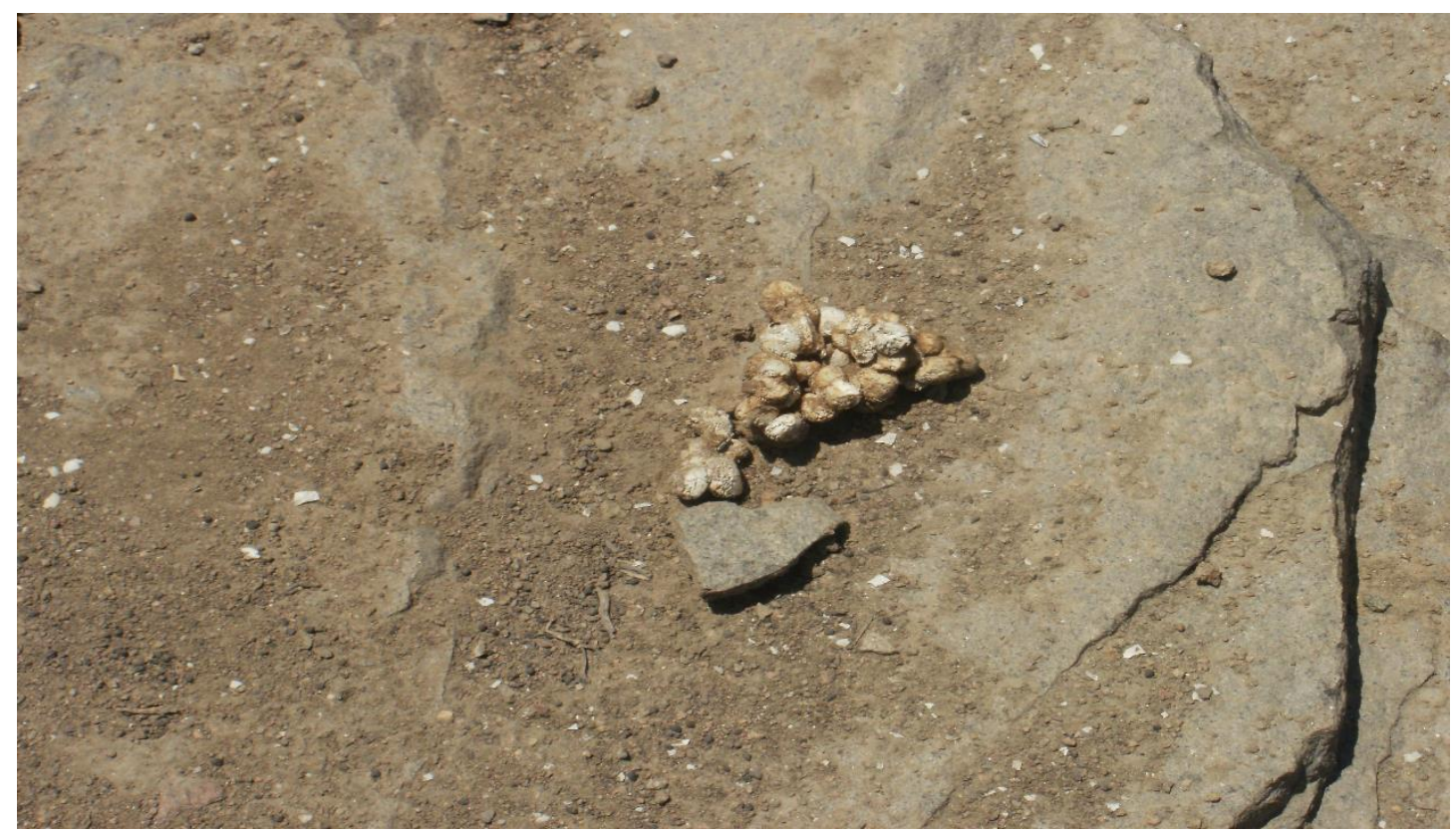

Figura 10. Excretas con semillas C. scabridum "sapote" de Lycalopex sechurae integrante de los tres niveles tróficos en el Área de Conservación Privada Lomas del Cerro Campana durante el 2019 